\title{
Humoral response to EBV is associated with cortical atrophy and lesion burden in patients with MS
}

\section{OPEN}

Robert Zivadinov, MD,

$\mathrm{PhD}$

Nicole Cerza, BS

Jesper Hagemeier, $\mathrm{PhD}$

Ellen Carl, MS

Darlene Badgett, MS

Deepa P. Ramasamy, MD

Bianca Weinstock-

Guttman, MD

Murali Ramanathan, $\mathrm{PhD}$

Correspondence to

Dr. Zivadinov:

rzivadinov@bnac.net

Supplemental data at Neurology.org/nn

\section{ABSTRACT}

Objective: Because dysregulated Epstein-Barr virus (EBV)-infected B cells may induce meningeal inflammation, which contributes to cortical pathology in multiple sclerosis (MS), we investigated associations between antibody responses to EBV and development of cortical pathology in MS.

Methods: We included 539 patients with MS (369 with relapsing-remitting MS, 135 with secondary progressive MS, and 35 with primary progressive MS), 66 patients with clinically isolated syndrome (CIS), 63 patients with other neurologic diseases (OND), and 178 age- and sex-matched healthy controls $(\mathrm{HC})$. All participants were scanned on 3T MRI. Serum samples were analyzed for lgG antibodies against EBV viral capsid antigen (VCA) and EBV nuclear antigen-1 (EBNA-1), and their quartiles were determined on the whole study sample. Differences between the study groups were assessed using analysis of covariance adjusted for multiple comparisons.

Results: More than $30 \%$ of patients with MS and CIS presented with the highest quartile of antiEBV-VCA and -EBNA-1 status compared to $\leq 10 \%$ of HC $(p<0.001)$. The figures were 9 $(14.3 \%)$ and $7(12.3 \%)$ for patients with OND. Patients with MS with the highest quartile of anti-EBV-VCA showed significantly increased T2 lesion volume $(p=0.001)$, T1 lesion number $(p=0.002)$, and T1 lesion volume $(p=0.04)$ and decreased gray matter $(p=0.041)$ and cortical $(p=0.043)$ volumes compared to patients with MS with lower quartiles. No significant differences of MRI outcomes in patients with CIS, patients with OND, and HC with lower or highest quartiles of anti-EBV-VCA and -EBNA-1 were detected.

Conclusions: Humoral response to anti-EBV-VCA and -EBNA-1 is associated with more advanced cortical atrophy, accumulation of chronic T1 black holes, and focal white matter lesions in patients with MS. Neurol Neuroimmunol Neuroinflamm 2016;3:e190; doi: 10.1212/NXI.0000000000000190

\section{GLOSSARY}

ANCOVA = analysis of covariance; $\mathbf{C E}=$ contrast-enhancing; $\mathbf{C I S}=$ clinically isolated syndrome; $\mathbf{C M V}=$ cytomegalovirus; EBNA-1 = Epstein-Barr virus nuclear antigen-1; EBV = Epstein-Barr virus; FLAIR = fluid-attenuated inversion recovery; $\mathbf{G M}=$ gray matter; $\mathbf{H C}=$ healthy controls; $\mathbf{L V}=$ lesion volume; $\mathbf{M S}=$ multiple sclerosis; $\mathbf{N B V}=$ normalized brain volume; $\mathbf{N C V}=$ normalized cortical volume; $\mathbf{N G M V}=$ normalized gray matter volume; $\mathbf{N L V V}=$ normalized lateral ventricle volume; NWMV = normalized white matter volume; OND = other neurologic diseases; PPMS = primary progressive MS; RRMS = relapsing-remitting MS; SE = spin-echo; SET study = Observational Study of Early Interferon beta 1-a Treatment in High Risk Subjects after CIS; SPMS = secondary progressive MS; TE = echo time; TI = inversion time; TR = repetition time; VCA $=$ viral capsid antigen; $\mathbf{W M}=$ white matter

One of the scientific challenges of investigating environmental risk factors in patients with multiple sclerosis (MS) is that they represent common exposures in the general population. For example, Epstein-Barr svirus (EBV) is a common environmental exposure and $>90 \%$ of the world's population is seropositive. ${ }^{1}$

In patients with clinically isolated syndrome (CIS), anti-EBV nuclear antigen-1 (EBNA-1) antibody levels are associated with progression to clinically definite MS and with the formation

From the Buffalo Neuroimaging Analysis Center (R.Z., D.B., M.R.) and Jacobs Multiple Sclerosis Center (B.W.-G.), Department of Neurology; MR Imaging Clinical Translational Research Center (R.Z., N.C., J.H., E.C., D.P.R., M.R.), School of Medicine and Biomedical Sciences, University at Buffalo, State University of New York, Buffalo, NY; and Department of Pharmaceutical Sciences (M.R.), State University of New York, Buffalo, NY.

Funding information and disclosures are provided at the end of the article. Go to Neurology.org/nn for full disclosure forms. The Article Processing Charge was paid by the authors.

This is an open access article distributed under the terms of the Creative Commons Attribution-NonCommercial-NoDerivatives License 4.0 (CC BY-NC-ND), which permits downloading and sharing the work provided it is properly cited. The work cannot be changed in any way or used commercially. 
of new inflammatory lesions. ${ }^{2-4}$ Crosssectional and longitudinal studies in patients with MS suggest that exposure to EBV may increase the likelihood of disease progression, as evidenced by clinical and MRI outcomes of disease severity. ${ }^{2-11}$

In the last decade, it has been established that cortical gray matter (GM) pathology is strongly associated with the presence of cortical subpial lesions, ${ }^{12}$ meningeal inflammation in the form of ectopic lymphoid-like structures, ${ }^{13}$ and retrograde wallerian degeneration of neurons. ${ }^{14}$ Clusters of meningeal inflammatory cells may act to sustain the intrathecal immune response and engender subpial cortical lesions. ${ }^{15,16}$ It has been hypothesized that dysregulated $\mathrm{EBV}$-infected $\mathrm{B}$ cells ${ }^{17}$ may induce meningeal inflammation that could contribute to GM pathology. Our previous work suggested that higher levels of EBV antibodies are associated with increased MRI lesion activity and greater brain atrophy, particularly of the GM. ${ }^{2,7-9}$

In this large cohort study, we aimed to examine the association of cortical pathology and IgG anti-EBV antibodies, as measured by cortical atrophy. We focused our attention on cortex because subpial cortical lesions and meningeal inflammation are emerging from pathology and imaging studies as the most important pathologic substrate for cognitive and physical disease progression in $\mathrm{MS}^{12,15,18,19}$ and EBV-infected B cells may play role in its pathogenesis. ${ }^{17,20}$

METHODS Study population. This study used baseline data from an ongoing prospective study of cardiovascular, environmental, and genetic risk factors in MS that enrolled more than 1,000 patients with CIS, MS, and other neurologic diseases (OND) and healthy controls (HC). ${ }^{21,22}$ The inclusion criteria for this substudy of the association of EBV and cytomegalovirus (CMV) and MRI outcomes were (1) having a valid serum sample for anti-EBV or anti-CMV antibody status determination, (2) having CIS, MS, or OND or being an HC, (3) being between 18 and 75 years old, and (4) having an MRI performed within 30 days of physical/neurologic/serum sample examination with the standardized study protocol. Exclusion criteria were presence of relapse and steroid treatment in the 30 days preceding study entry for patients with CIS and MS, preexisting medical conditions known to be associated with brain pathology (cerebrovascular disease, positive history of alcohol abuse), and pregnancy.

All participants had a physical and neurologic examination. $\mathrm{HC}$ needed to meet the health screen MRI requirements and had to have a normal neurologic examination. They were recruited from hospital personnel and respondents to a local advertisement or were spouses of the patients with MS. Race/ethnicity was determined according to US Census Bureau definitions.

Standard protocol approvals, registrations, and patient consents. The study protocol was approved by the local institutional review board and all participants gave their written informed consent.

Determination of anti-EBV and anti-CMV antibodies. The technicians conducting analyses of anti-EBV and anti-CMV antibodies were blinded to the disease and clinical group status. ELISA kits from Diamedix Corporation (Miami, FL) were used to quantify anti-EBV viral capsid antigen (VCA), EBNA-1 $\mathrm{IgG}$, and anti-CMV antibodies, as previously described. ${ }^{2,7}$

MRI acquisition and analysis. All participants were examined on a 3T GE Signa Excite HD 12.0 Twin Speed 8-channel scanner (General Electric, Milwaukee, WI). MRI sequences included the axial dual fast spin-echo (SE) T2/proton density-weighted image, 3D spoiled gradient-recalled T1-weighted image, SE T1weighted image pre- and postcontrast, and fluid-attenuated inversion recovery (FLAIR) scans. Pulse sequence characteristics for 3T MRI were as follows. All scans were acquired with a $256 \times$ 256 matrix and a 25.6-cm field of view (FOV) for an in-plane resolution of $1 \times 1 \mathrm{~mm}^{2}$ with a phase FOV of $75 \%$ and one average. Sequence-specific parameters were as follows. For the T2/ proton density-weighted image: 3-mm-thick slices with no gap, TE1 (echo time)/TE2/TR (repetition time) $=12 / 95 / 3,000$ milliseconds, echo train length $=14$, flip angle $=90^{\circ}$; for the FLAIR scans: 3-mm-thick slices with no gap, TE/TI (inversion time $) / T R=120 / 2,100 / 8,500$ milliseconds, flip angle $=90^{\circ}$; for 3D T1-weighted image: 1-mm-thick slices with no gap, TE/TI/ $\mathrm{TR}=2.8 / 900 / 5.9$ milliseconds, flip angle $=10^{\circ}$; and for $\mathrm{SE}$ T1-weighted image: 3-mm-thick slices with no gap, TE/TR = $16 / 600$ milliseconds, flip angle $=90^{\circ}$. The SE T1-weighted image sequence was obtained after injection of a single-dose IV bolus $(0.1$ $\mathrm{mMol} / \mathrm{kg}$ gadolinium-diethylenetriaminepentacetate) 5 minutes after administration of contrast agent only in patients with MS and CIS.

MRI analysts were blinded to the participants' physical and neurologic condition. The MRI measures included in the analysis were contrast-enhancing (CE) T1 and T2 lesion number and lesion volumes (LVs), assessed by a semi-automated edge detection contouring/thresholding technique, ${ }^{23}$ and measures of brain atrophy, including normalized brain volume (NBV), normalized GM volume (NGMV), normalized cortical volume (NCV), normalized white matter (WM) volume (NWMV), and normalized lateral ventricle volume (NLVV), ${ }^{23}$ assessed by the SIENAX 2.6 cross-sectional software tool. ${ }^{24}$

Statistical analysis. Statistical analysis was performed using SPSS version 21.0 (IBM, Armonk, NY). The data were investigated separately by disease group (HC, CIS, MS, and OND) and by MS disease subtype (relapsing-remitting MS [RRMS], secondary progressive MS [SPMS], and primary progressive MS [PPMS]).

The analyses were focused on MRI associations with antiEBV antibody levels rather than antibody positivity, because the percentage of participants positive for anti-EBV-VCA and anti-EBV-EBNA-1 antibodies was very high (>99\%) because of the ubiquity of exposure to EBV. Given that relative concentrations of the anti-VCA and anti-EBNA-1 antibodies are not normally distributed, these were categorized into lower and the highest quartiles based on the whole study sample regardless of clinical group, as previously reported. ${ }^{2,7}$ We also performed a 
Pearson correlation analysis between anti-EBV-VCA, anti-EBVEBNA-1, and anti-CMV antibodies and MRI outcomes.

Differences in the categorical variables between the groups were analyzed using the $\chi^{2}$ test. An analysis of variance was used to analyze the differences in continuous variables between the groups. The differences in ordinal variables between the groups were assessed using nonparametric statistics. We used analysis of covariance (ANCOVA), adjusted for age, sex, and treatment status, to investigate MRI differences between anti-EBV-VCA and anti-VCA-EBNA-1 highest and lower quartiles and anti-CMV positive and negative status groups. Given the high colinearity between age and disease duration, the latter was not used as a covariate.

The association analyses between anti-EBV and anti-CMV antibodies and MRI outcomes were corrected for multiple testing using the Benjamini-Hochberg correction. ${ }^{25}$ Nominal $p$ values $<0.05$ were regarded as significant, using 2 -tailed testing.

RESULTS Demographic and clinical characteristics of the study groups. Demographic and clinical characteristics of the study groups are shown in table 1 . A total of 846 consecutive participants who fulfilled inclusion and exclusion criteria entered this substudy of EBV and CMV and MRI outcomes in MS. The study population consisted of $178 \mathrm{HC}$, 539 patients with MS, 66 patients with CIS, and 63 patients with OND (25 with neurodegenerative, 18 with autoimmune, 10 with vascular, and 10 with neuromuscular origin). Patients with MS, HC, and patients with OND were age- and sex-matched, whereas patients with CIS were significantly younger, had shorter disease duration, and had a lower level of disability, as expected (table 1 and table e-1 at Neurology.org/nn).

Demographic and clinical characteristics of the patients with MS by subtype are shown in table 2 . There were 368 patients with RRMS, 135 patients with SPMS, and 35 patients with PPMS in the study. As expected, patients with SPMS and PPMS were significantly older, had longer disease duration, and had a higher level of disability than those with RRMS.

Eighteen (10.1\%) HC, 7 (12.3\%) patients with OND, 68 (31.2\%) patients with MS, and 22 (33.3\%) patients with CIS were grouped into the highest quartile of anti-EBV-VCA antibodies; the corresponding numbers for anti-EBV-EBNA-1 antibodies were $13(7.3 \%), 9$ (14.3\%), 182 (33.8\%), and 19 (28.8\%), respectively (table 1 and table e-1). The mean titer of anti-EBV-VCA antibodies was significantly greater in patients with MS and CIS than in HC (table $1, p<0.001$ ), and there was a trend for greater mean titer of anti-EBVEBNA-1 antibodies in patients with MS and CIS (table $1, p=0.068$ ). Frequency of the highest quartile status group of anti-EBV-VCA antibodies was similarly distributed across all MS subtypes. In contrast, more patients with RRMS (36.3\%) than those with SPMS (22.2\%) and PPMS (28.6\%) were found in the highest quartile group of anti-EBV-EBNA-1 antibodies $(p=0.001)$. No significant differences in mean titer of anti-EBV-VCA and -EBNA-1 antibodies were detected between different MS subtypes.

No significant differences between patients with MS and CIS and HC (table 1) or between different OND subtypes (table e-1) were found for anti-CMV antibody positivity or mean titer.

MRI differences according to the anti-EBV-VCA antibodies. Table 3 and figure 1 show group differences of MRI measures between the anti-EBV-VCA highest and lower quartiles across the study groups. Among patients with MS, increased T2 LV ( $p=$ $0.001)$, T1 lesion number $(p=0.002)$ and $\operatorname{LV}(p=$ $0.04)$ and decreased NGMV $(p=0.041)$ and NCV $(p=0.043)$ were observed in the highest quartile status group. There were no differences between the antiEBV-VCA highest and lower quartile status groups and MRI outcomes in the HC, OND, or CIS study groups. In Pearson correlation analysis performed in patients with MS, there was a significant association between the greater anti-EBV-VCA titer and increased T1 lesion number $(r=0.12, p=0.008)$ and decreased $\operatorname{NGMV}(r=0.11, p=0.014)$ and NCV $(r=0.1$, $p=0.018)$. No associations between the anti-EBVVCA titers and MRI outcomes were detected in HC, OND, or CIS study groups.

Group differences of MRI outcomes between antiEBV-VCA highest and lower quartile status groups within RRMS, SPMS, and PPMS subtypes are shown in table 4 and figure 1. An increased T2 LV ( $p=$ $0.018)$ and T1 lesion number $(p=0.039)$ and decreased NGMV $(p=0.01)$ and $\operatorname{NCV}(p=$ $0.025)$ were detected in patients with RRMS in the highest quartile status group. Patients with SPMS showed increased T2 LV ( $p=0.004)$, T1 lesion number $(p=0.017)$, and LV $(p=0.033)$. No anti-EBVVCA highest and lower quartile status group MRI outcome differences were found for the patients with PPMS. In Pearson correlation analysis performed in patients with RRMS, there was a significant association between the greater anti-EBV-VCA titer and decreased $\operatorname{NGMV}(r=0.13, p=0.019)$ and NCV $(r=0.12$, $p=0.034)$. Patients with SPMS showed a significant association between increased T2 LV and greater antiEBV-VCA titer $(r=0.22, p=0.011)$. No associations between the anti-EBV-VCA titers and MRI outcomes were detected in patients with PPMS.

MRI differences according to the anti-EBV-EBNA-1 antibodies. Similar within-group analyses were carried out for anti-EBV-EBNA-1 antibodies. No anti-EBV-EBNA-1 highest and lower quartile status group MRI outcome differences were found for patients with MS, CIS, and OND and $\mathrm{HC}$ (table e-2 and figure e-1). In Pearson 
Table 1 Demographic and clinical characteristics of the study groups

\begin{tabular}{|c|c|c|c|c|}
\hline & $\mathrm{HC}(\mathrm{n}=178)$ & MS $(n=539)$ & $\operatorname{CIS}(n=66)$ & $p$ Value \\
\hline Female, n (\%) & $121(68)$ & $367(68.1)$ & $49(74.2)$ & 0.586 \\
\hline Age, y, mean (SD) & $43.5(15.7)$ & 45.7 (12.2) & 39.7 (11.0) & 0.001 \\
\hline Age at onset, $y$, mean (SD) & NA & $31.5(10.4)$ & 35.2 (10.9) & 0.008 \\
\hline Disease duration, $y$, mean (SD) & NA & $14.4(10.7)$ & $1.9(2.0)$ & $<0.001$ \\
\hline Presence of DMT, $n$ (\%) & NA & 381 (70.7) & $30(45.5)$ & $<0.001$ \\
\hline Interferon $\beta-1 a$ & & 175 (32.5) & $23(76.7)$ & \\
\hline Glatiramer acetate & & $110(20.5)$ & 7 (23.3) & \\
\hline Natalizumab & & $76(14.1)$ & & \\
\hline Mycophenolate mofetil & & $7(1.3)$ & & \\
\hline IV immnuglobulin & & $6(1.1)$ & & \\
\hline Azathioprine & & $4(0.7)$ & & \\
\hline Mitoxantrone & & $3(0.6)$ & & \\
\hline EDSS score, median (IQR) & NA & $3.0(1.5-5.5)$ & $1.5(1.0-2.0)$ & $<0.001$ \\
\hline \multicolumn{5}{|l|}{ Race, n (\%) } \\
\hline White & $155(87)$ & $507(94)$ & $61(92.4)$ & 0.078 \\
\hline Hispanic/Latino & $2(1.1)$ & $8(1.5)$ & 2 (3) & \\
\hline Black/African American & $15(8.4)$ & $20(3.7)$ & 2 (3) & \\
\hline Asian & $3(1.7)$ & $2(0.4)$ & $1(1.6)$ & \\
\hline Other & $3(1.7)$ & $2(0.4)$ & 0 & \\
\hline \multicolumn{5}{|l|}{$\begin{array}{l}\text { Anti-EBV-VCA, highest vs lower } \\
\text { quartiles, } \mathrm{n}(\%)\end{array}$} \\
\hline Lower quartiles & $160(89.9)$ & 371 (68.8) & $44(66.7)$ & $<0.001$ \\
\hline Highest quartile & $18(10.1)$ & 168 (31.2) & 22 (33.3) & \\
\hline \multicolumn{5}{|l|}{$\begin{array}{l}\text { Anti-EBV-EBNA-1, highest vs lower } \\
\text { quartiles, } \mathrm{n}(\%)\end{array}$} \\
\hline Lower quartiles & $165(92.7)$ & 357 (66.2) & 47 (71.2) & $<0.001$ \\
\hline Highest quartile & $13(7.3)$ & $182(33.8)$ & $19(28.8)$ & \\
\hline \multicolumn{5}{|l|}{ Anti-EBV titer, mean (SD) median } \\
\hline Anti-EBV-VCA & $74.7(46.3) 112.1$ & $150(96.1) 171$ & $140(95.4) 155$ & $<0.001$ \\
\hline Anti-EBV-EBNA-1 & $114.7(27.5) 487.1$ & 262.7 (131) 805 & $212.2(103.5) 433.4$ & 0.068 \\
\hline \multicolumn{5}{|l|}{ Anti-CMV, n (\%) } \\
\hline Negative & $107(60.1)$ & $311(57.7)$ & $37(56.1)$ & 0.838 \\
\hline Positive & 71 (39.9) & 228 (42.3) & 29 (43.9) & \\
\hline Anti-CMV titer, mean (SD) median & $230(664) 0$ & $355(1,040) 0$ & $237.6(553.7) 0$ & 0.231 \\
\hline
\end{tabular}

Abbreviations: CIS = clinically isolated syndrome; CMV = cytomegalovirus; DMT = disease-modifying therapy; EBNA-1 = Epstein-Barr virus nuclear antigen-1; EBV = Epstein-Barr virus; EDSS = Expanded Disability Status Scale; HC = healthy controls; IQR = interquartile range; MS = multiple sclerosis; NA = not available; VCA = viral capsid antigen. Differences between the groups were tested using $\chi^{2}$ test, Mann-Whitney rank-sum, or analysis of variance.

correlation analysis, no associations between the antiEBV-EBNA-1 titers and MRI outcomes were detected in MS, HC, OND, or CIS study groups.

Table e-3 and figure e-1 show anti-EBV-EBNA-1 highest vs lower quartile status group MRI outcome differences for MS subtypes. Patients with RRMS in the anti-EBV-EBNA-1 highest quartile status group showed increased T1 lesion number $(p=0.035)$ and decreased NGMV $(p=0.008)$ and NCV $(p=0.035)$.
Patients with PPMS in the anti-EBV-EBNA-1 highest quartile status group showed increased T2 lesion number $(p=0.047)$. In Pearson correlation analysis, there was a trend for decreased NGMV $(p=0.052)$, NCV $(p=0.074)$, and T1 lesion number $(p=0.079)$ in patients with RRMS. No anti-EBV-EBNA-1 highest and lower quartile status group MRI outcome differences were found for the patients with SPMS. In Pearson correlation analysis, no associations between the 


\begin{tabular}{|c|c|c|c|c|}
\hline \multirow[t]{2}{*}{ Demographic an } & characteristics of $t$ & patients with MS & & \multirow[b]{2}{*}{$p$ Value } \\
\hline & RRMS ( $n=369$ ) & SPMS $(n=135)$ & PPMS (n = 35) & \\
\hline Female, n (\%) & 229 (62.1) & 99 (73.3) & $21(60)$ & 0.228 \\
\hline Age, y, mean (SD) & 43.9 (10.2) & $53.2(8.4)$ & $54.4(6.6)$ & $<0.001$ \\
\hline Age at onset, $y$, mean (SD) & $31.7(9.1)$ & $31.7(10.8)$ & $39.4(9.7)$ & $<0.001$ \\
\hline Disease duration, $y$, mean (SD) & $6.2(7.8)$ & $12.1(8.8)$ & $22.1(11.7)$ & $<0.001$ \\
\hline Presence of DMT, $\mathrm{n}(\%)$ & 269 (72.9) & $96(71.1)$ & $16(45.7)$ & 0.003 \\
\hline Interferon $\beta-1 a$ & $126(34.1)$ & $41(30.4)$ & 8 (22.3) & \\
\hline Glatiramer acetate & $78(21.1)$ & 26 (19.3) & $6(17.1)$ & \\
\hline Natalizumab & $57(15.5)$ & $19(14.1)$ & $0(0)$ & \\
\hline Mycophenolate mofetil & $3(0.8)$ & $3(2.2)$ & $1(6.3)$ & \\
\hline IV immnuglobulin & $4(1.1)$ & $1(0.7)$ & $1(6.3)$ & \\
\hline Azathioprine & $0(0)$ & 4 (3) & $0(0)$ & \\
\hline Mitoxantrone & $1(0.3)$ & $2(1.4)$ & $0(0)$ & \\
\hline EDSS score, median (IQR) & $2.0(1.5-3.0)$ & $6.0(4.5-6.5)$ & $6.0(3.9-6.5)$ & $<0.001$ \\
\hline \multicolumn{5}{|l|}{ Race, n (\%) } \\
\hline White & 345 (93.5) & $130(96.6)$ & 32 (93.8) & 0.505 \\
\hline Hispanic/Latino & $6(1.9)$ & $1(0.8)$ & $1(3.1)$ & \\
\hline Black/African American & $15(4.8)$ & $3(1.7)$ & 2 (3.1) & \\
\hline Asian & $2(0.6)$ & 0 & 0 & \\
\hline Other & $1(0.3)$ & $1(0.8)$ & 0 & \\
\hline \multicolumn{5}{|l|}{$\begin{array}{l}\text { Anti-EBV-VCA, highest vs lower } \\
\text { quartiles, } \mathrm{n}(\%)\end{array}$} \\
\hline Lower quartiles & 261 (70.7) & $87(64.4)$ & $27(77.1)$ & 0.344 \\
\hline Highest quartile & 108 (29.3) & 48 (35.6) & 8 (22.9) & \\
\hline \multicolumn{5}{|c|}{$\begin{array}{l}\text { Anti-EBV-EBNA-1, highest vs lower } \\
\text { quartiles, } n(\%)\end{array}$} \\
\hline Lower quartiles & $235(63.7)$ & 105 (77.8) & $25(71.4)$ & 0.001 \\
\hline Highest quartile & 134 (36.3) & 30 (22.2) & $10(28.6)$ & \\
\hline \multicolumn{5}{|l|}{ Anti-EBV titer, mean (SD) median } \\
\hline Anti-EBV-VCA & $153.6(97.1) 177.6$ & $151.2(108.9) 137.2$ & $155.8(85.1) 240.7$ & 0.986 \\
\hline Anti-EBV-EBNA-1 & 307.3 (153.3) 989.7 & $151.2(93) 163.6$ & $227.2(94.5) 362.4$ & 0.169 \\
\hline
\end{tabular}

Abbreviations: DMT = disease-modifying therapy; EBNA-1 = Epstein-Barr virus nuclear antigen-1; EBV = Epstein-Barr virus; EDSS = Expanded Disability Status Scale; IQR = interquartile range; MS = multiple sclerosis; PPMS = primary progressive MS; RRMS = relapsing-remitting MS; SPMS = secondary progressive MS; VCA = viral capsid antigen. Differences between the groups were tested using $\chi^{2}$ test, Kruskall-Wallis, or analysis of variance.

anti-EB- EBNA-1 titers and MRI outcomes were detected in patients with SPMS or PPMS.

MRI differences according to the anti-CMV antibodies. No significant associations between MRI outcomes and anti-CMV antibody positivity or mean titer were found in patients with MS, CIS, and OND or in HC. Because no significant results were found in the MS group, no further analyses regarding MS subtypes were performed.

DISCUSSION This cohort study examined association between the humoral response to EBV and MRI outcomes and investigated specifically the effect of this relationship in the cortex of patients with MS in vivo. Moreover, this study investigated whether the association of humoral response to EBV and MRI outcomes is MS-specific. The present study included 63 patients with OND and $178 \mathrm{HC}$ who were age- and sex-matched to the patients with MS and who underwent anti-EBV and anti-CMV antibody determination and standardized 3T MRI with identical study procedures as part of a prospective cardiovascular, environmental, and genetic risk factor study in MS. ${ }^{21,22}$ We did not find significant associations between humoral response to EBV and CMV and MRI outcomes in patients with OND (even of autoimmune origin) and 


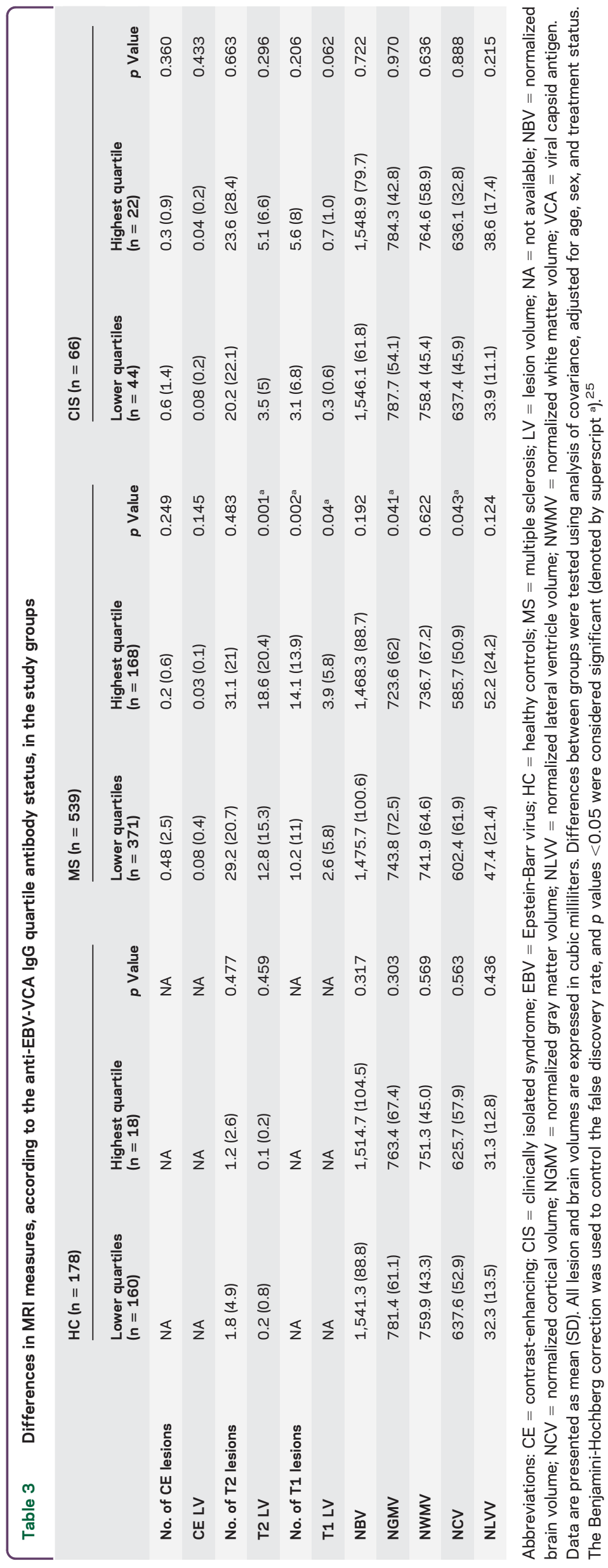

$\mathrm{HC}$, indicating that this relationship is MS-specific. Therefore, the current study supports a number of previous pathologic reports suggesting that meningeal inflammation and increased GM pathology may be induced by dysregulated EBVinfected B cells. ${ }^{13,17,20}$

It is well known that mononucleosis, an infection caused by EBV, has been linked to the increased risk of developing MS in susceptible adolescents and young adults. ${ }^{26}$ Evidence is mounting that EBV exerts its adverse disease susceptibility effects in MS via interactions with other host-related environmental and genetic factors. ${ }^{8,11,27}$ Elevated titers have been reported against both the EBV-VCA, which is expressed in the viral replicative cycle, and the antiEBV-EBNA-1, which is expressed in latently infected B lymphocytes. ${ }^{1}$ Anti-EBV-VCA and -EBNA-1 elevation may indicate a more severe infection among patients with MS. It has been shown that EBV infection-mediated $\mathrm{T}$ cell response could lead to cross-reactivity with self-antigens. There is an expansion of activated circulating $T$ cells that are specific for EBV latent and lytic antigens in patients with acute infectious mononucleosis. ${ }^{1}$

The association of EBV-infected B cells located in ectopic follicles ${ }^{13,17,20}$ and cortical pathology in patients with MS could not be initially replicated..$^{28,29}$ However, more-recent investigations that used sensitive radioactive probes against EBV-encoded RNA have provided further evidence consistent with the presence of EBV-infected cells that are colocalized with innate immune responses characterized by interferon $\alpha$ production in the brain of patients with MS. ${ }^{30}$ Higher frequency of $\mathrm{CD} 8^{+} \mathrm{T}$ cell response to latent and lytic EBV proteins in inactive, untreated patients with RRMS suggests that inability to control EBV infection during inactive MS could set the stage for intracerebral viral reactivation and disease progression. ${ }^{31}$ Another study found cells expressing EBVencoded small RNA of early lytic EBV proteins in the cortical lesion infiltrates of patients with SPMS. ${ }^{32}$

The results of the current study support the hypothesis that EBV-infected B cells may play an important role in the cortical and GM pathology. In fact, we found that the predilection of the brain volume loss in patients with MS was localized specifically to the cortex, as no significant differences were found between patients with MS for the highest and lower quartiles and relative mean titers of anti-EBVVCA and -EBNA-1 antibodies for the whole brain, WM, or lateral ventricles. Lesions in the GM are frequently recognized in the earliest phases of $\mathrm{MS}^{33}$ and in patients with radiologically isolated syndrome ${ }^{34}$ and could be related to meningeal inflammation and germinal centers in which anti-EBV antibody levels may play an important role. In a previous study, 
A

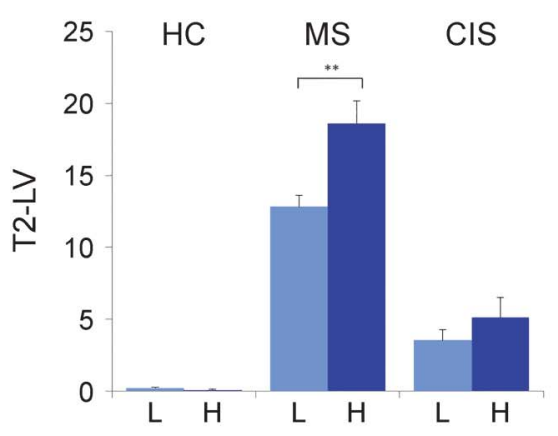

C

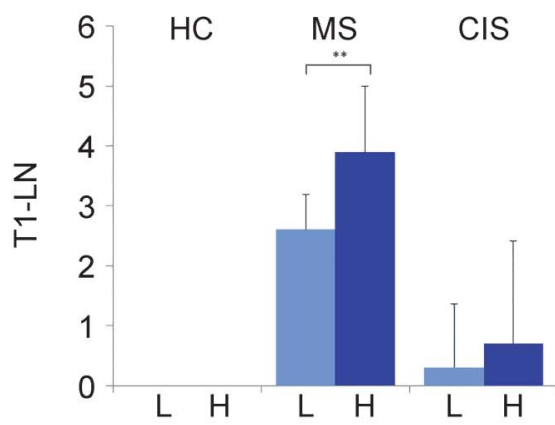

\section{B}

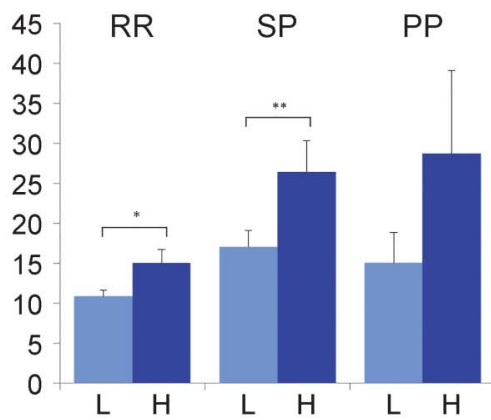

D

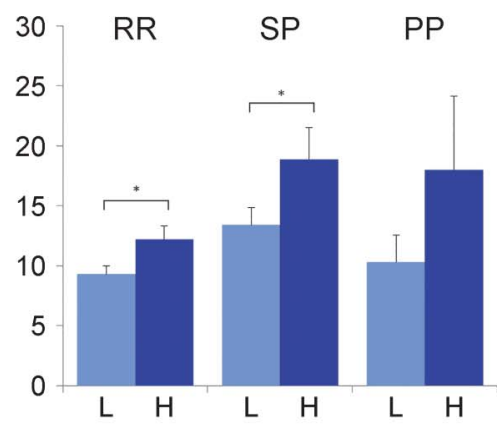

$\mathrm{E}$

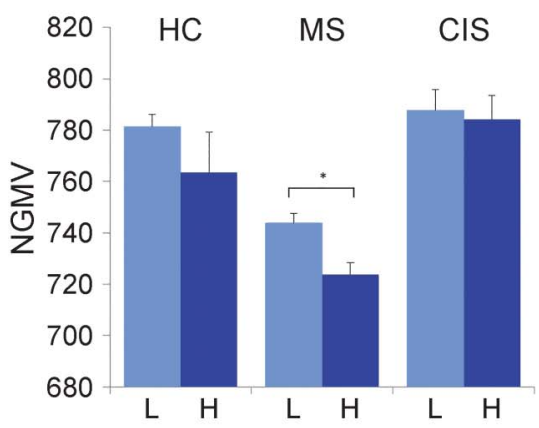

G

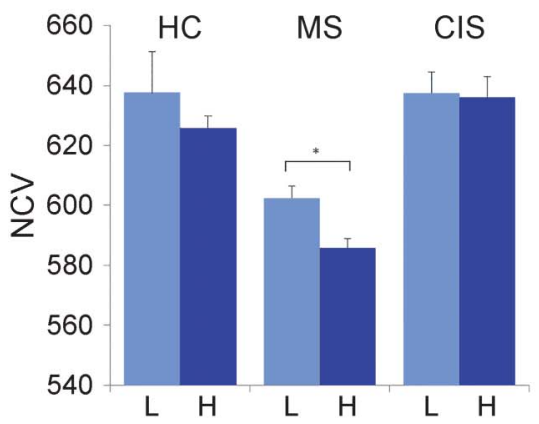

$\mathrm{F}$

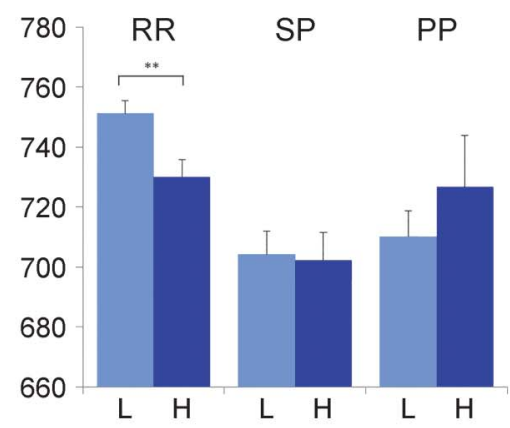

$\mathrm{H}$

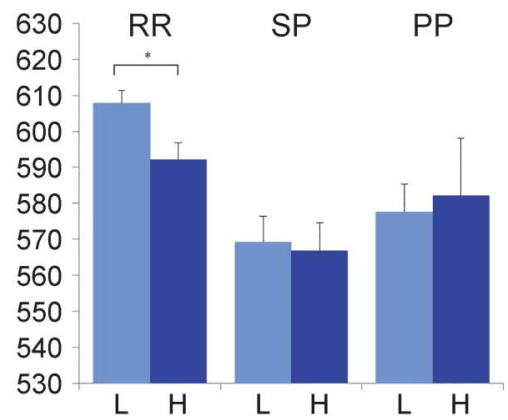

Data are presented as mean and SEM. All lesion and brain volumes are expressed in cubic milliliters. The data are displayed for healthy controls $(\mathrm{HC})$ and patients with multiple sclerosis (MS) and clinically isolated syndrome (CIS) on the left and for patients with relapsing-remitting MS (RR), secondary progressive MS (SP), and primary progressive MS (PP) on the right for T2 lesion volume (LV) (A, B), T1 LV (C, D), normalized gray matter volume (NGMV) (E, F), and normalized cortical volume (NCV) $(G, H) . H=$ highest quartile; $L=$ lower quartile. ${ }^{*} p<0.05 ;{ }^{*} p<0.01$.

we reported that GM atrophy was associated with anti-EBV-VCA antibody status in a sample of patients with MS recruited from Italy. ${ }^{9}$ The present study confirms these preliminary findings in a 5fold greater cohort of patients with MS who had different genetic and environment characteristics. It also 


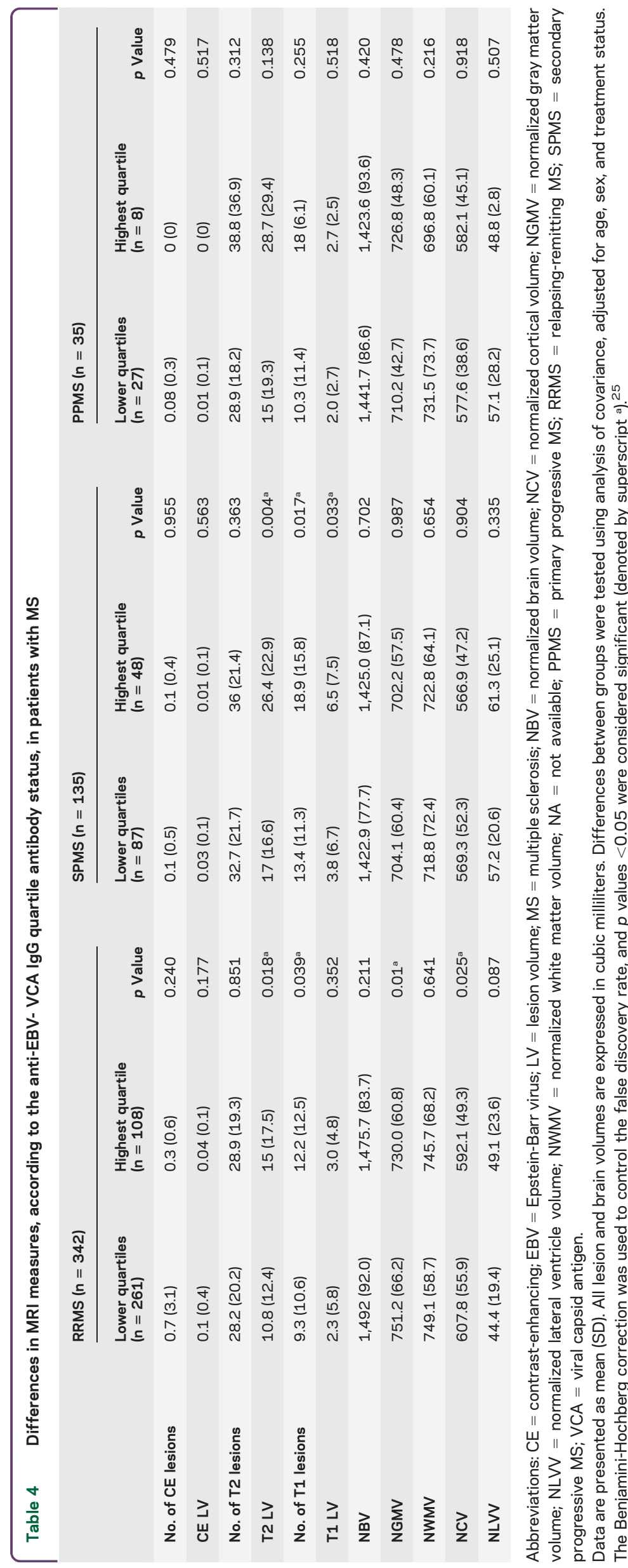

extends our initial observations ${ }^{9}$ by demonstrating that the humoral response to EBV has particular implications for the cortical pathology in MS. When the data were analyzed according to the MS disease subtypes, we found significant associations between cortical atrophy and anti-EBV-VCA and -EBNA-1 antibody status in RRMS but not in progressive MS subtypes, probably because of the lower sample size in these groups, especially in the PPMS group. This is in line with findings from a multicenter, prospective, longitudinal Observational Study of Early Interferon beta 1-a Treatment in High Risk Subjects after CIS (SET study), which showed that highest quartile antibody status of anti-EBV-VCA was associated with greater decrease in thalamus volume $(p=0.003)$ and a trend for decrease in cortical volume $(p=$ 0.028 ) over 2 years in 193 patients with CIS. ${ }^{7}$ In the same SET study, development of thalamic atrophy was better associated with development of clinically definite MS than accumulation of new T2 and CE lesions over 2 years. ${ }^{35}$ Therefore, our data suggest that humoral response to EBV may exert a robust effect in earlier stages of the MS disease process.

A retrospective analysis of 147 patients with CIS with 5-year follow-up in 117 patients with MS found that anti-EBV-EBNA-1 and -VCA antibody status immune responses were associated with baseline number of $\mathrm{T} 2$ lesions and with accumulation of new T2 lesions over 1 and 5 years. ${ }^{3}$ In a group of 50 patients with CIS, 25 patients with RRMS, and 25 patients with PPMS followed over a 5-year period, accumulation of CE lesions in patients with established MS was associated with increased titer of anti-EBV-EBNA-1 antibodies. ${ }^{4}$ In the SET study, the cumulative number of $\mathrm{CE}$ and $\mathrm{T} 2$ lesions during the 2-year period was greater for individuals in the highest quartile of anti-EBV-VCA antibodies. ${ }^{2}$ In our Italian MS EBV study, we found that anti-EBV-VCA antibody status was associated with higher T2 and T1 LV. ${ }^{9}$ In the present study, there was a significant association between increased $\mathrm{T} 2$ LV and number and volume of T1 lesions and antiEBV-VCA and -EBNA-1 antibody highest quartile status and anti-EBV-VCA titer. It is interesting that these associations were present in patients with both relapsing and chronic progressive MS, indicating that humoral response to EBV is associated with more advanced pathologic substrate in the MS lesions. The reactivation of EBV over long-term can be a source of antigenic challenge to the immune system that is capable of promoting the proliferation of $\mathrm{B}$ cells and also causing activation of autoreactive $\mathrm{T}$ cells, ${ }^{3}$ which can potentially explain increased $\mathrm{T} 2$ and $\mathrm{T} 1 \mathrm{LVs}$ in patients with the highest quartiles of anti-EBV-VCA and -EBNA-1. 
In order to determine whether antibody responses to another nearly ubiquitous virus (anti-CMV) are unique to patients with MS and CIS, we performed an additional analysis exploring anti-CMV positivity and mean titer differences between the study groups. No anti-CMV positivity or mean titer differences were found between patients with MS, CIS, and OND and HC. No associations between MRI outcomes and anti-CMV variables were found in any study group. These findings strengthen our results, indicating that humoral response to anti-EBV antibodies is MS-specific.

This study used an established, standardized method for determination of curve and the concentration of anti-EBV-VCA, anti-EBV-EBNA-1, and anti-CMV antibodies. ${ }^{2,7}$ We used quartiles for antiEBV antibody titers and concentration determination, as they are not normally distributed, or anti-CMV positivity in the statistical analyses. ${ }^{2,7}$ However, we also provided mean titer data and conducted Pearson correlation analyses between anti-EBV-VCA, anti-EBV-EBNA-1, and anti-CMV antibodies and MRI outcomes to allow comparison with the previous literature. The ANCOVA and Pearson correlation analyses for the anti-EBV-VCA antibody status and MRI outcomes yielded similar results. However, there were discrepancies between ANCOVA and Pearson correlation results for the anti-EBV-EBNA-1 antibodies and MRI outcomes in patients with RRMS, possibly due to antibody values that were not normally distributed, which could have affected the correlation results.

The methodologic strengths of this study are that EBV and CMV examinations were carried out by expert physicians on a large cohort of $\mathrm{HC}$ and patients with MS and CIS in the setting of a specialized MS center and that MRIs was collected with standardized and sophisticated imaging protocol at 3T. However, the study is not without limitations. Use of more specific MRI sequences such as double inversion recovery could have increased the ability to capture part of lesion GM pathology in vivo. ${ }^{19}$ The cross-sectional nature of this study cannot establish a causal relationship between EBV and greater cortical atrophy in patients with MS, although findings from the control groups indicated that these associations are MS-specific. A longitudinal 5-year examination of the present cohort is under way in our center to determine the cause-effect relationship of humoral response to EBV and progression of MRI and clinical outcomes.

Our results demonstrate that humoral immune responses to herpesviruses such as EBV are associated with more advanced cortical atrophy and accumulation of chronic T1 black holes and focal WM lesions in patients with MS. The results provide evidence for further research aimed at modulating the response of patients with MS to these viruses via drug and vaccine strategies.

\section{AUTHOR CONTRIBUTIONS}

Robert Zivadinov, Bianca Weinstock-Guttman, and Murali Ramanathan substantially contributed to the concept and design of the study. Robert Zivadinov drafted the article, and all authors revised it critically for important intellectual content. Nicole Cerza and Jesper Hagemeier performed statistical analysis. All authors had access to the data.

\section{STUDY FUNDING}

This study was funded by internal resources of the Buffalo Neuroimaging Analysis Center and Department of Neurology, University of Buffalo. In addition, we received support from the National Multiple Sclerosis Society (RG4836A5, RR2007A2) and Department of Defense (MS090122).

\section{DISCLOSURE}

R. Zivadinov served on the scientific advisory board for Genzyme and Novartis; received speaker honoraria from Biogen Idec, Claret, EMD Serono, Inc, Novartis, Sanofi-Genzyme, and Teva; has consulted for Biogen, Claret, EMD Serono, Inc, Novartis, Sanofi-Genzyme, and Teva; and received research support from Biogen Idec, Claret, EMD Serono, Inc, Novartis, Sanofi-Genzyme, and Teva. N. Cerza, J. Hagemeier, E. Carl, D. Badgett, and D.P. Ramasamy report no disclosures. B. Weinstock-Guttman served on the scientific advisory board for National Multiple Sclerosis Society; served on the advisory board and speaker engagements for Biogen, Teva, EMD Serono, Pfizer, Novartis, Acorda, Genzyme \& Sanofi, and Questcor; is on the editorial board for Multiple Sclerosis International, BMJ Neurology, and Journal of Multiple Sclerosis; has consulted for Biogen, Teva, EMD Serono, Novartis, Genentech, Questcor, and Genzyme \& Sanofi; and received research support from Biogen, EMD Serono, Teva, Novartis, Genzyme \& Sanofi, Questcor, NIH, NMSS, DOD, National MS Society, and Clinical grant for Pediatric MS Center of Excellence DOD. M. Ramanathan is on the editorial board for AAPS Journal; receives publishing royalties from Pinnacle, Summit, and Zenith; and received research support from Serono, Novartis, Pfizer, Biogen, Department of Defense, and National Multiple Sclerosis Society. Go to Neurology.org/nn for full disclosures.

Received May 11, 2015. Accepted in final form October 30, 2015.

\section{REFERENCES}

1. Lucas RM, Hughes AM, Lay ML, et al. Epstein-Barr virus and multiple sclerosis. J Neurol Neurosurg Psychiatry 2011;82:1142-1148.

2. Horakova D, Zivadinov R, Weinstock-Guttman B, et al. Environmental factors associated with disease progression after the first demyelinating event: results from the multicenter SET study. PLoS One 2013;8:e53996.

3. Lunemann JD, Tintore M, Messmer B, et al. Elevated Epstein-Barr virus-encoded nuclear antigen-1 immune responses predict conversion to multiple sclerosis. Ann Neurol 2010;67:159-169.

4. Farrell RA, Antony D, Wall GR, et al. Humoral immune response to EBV in multiple sclerosis is associated with disease activity on MRI. Neurology 2009;73:32-38.

5. Buljevac D, van Doornum GJ, Flach HZ, et al. EpsteinBarr virus and disease activity in multiple sclerosis. J Neurol Neurosurg Psychiatry 2005;76:1377-1381.

6. Wandinger K, Jabs W, Siekhaus A, et al. Association between clinical disease activity and Epstein-Barr virus reactivation in MS. Neurology 2000;55:178-184.

7. Zivadinov R, Chin J, Horakova D, et al. Humoral responses to herpesviruses are associated with neurodegeneration after a demyelinating event: results from the multi-center set study. J Neuroimmunol 2014;273:58-64. 
8. Zivadinov R, Weinstock-Guttman B, Zorzon M, et al. Gene-environment interactions between HLA B7/A2, EBV antibodies are associated with MRI injury in multiple sclerosis. J Neuroimmunol 2009;209:123-130.

9. Zivadinov R, Zorzon M, Weinstock-Guttman B, et al. Epstein-Barr virus is associated with grey matter atrophy in multiple sclerosis. J Neurol Neurosurg Psychiatry 2009; 80:620-625.

10. Kvistad S, Myhr KM, Holmøy T, et al. Antibodies to Epstein-Barr virus and MRI disease activity in multiple sclerosis. Mult Scler 2014;20:1833-1840.

11. Weinstock-Guttman B, Horakova D, Zivadinov R, et al. Interactions of serum cholesterol with anti-herpesvirus responses affect disease progression in clinically isolated syndromes. J Neuroimmunol 2013;263:121-127.

12. Kutzelnigg A, Lucchinetti CF, Stadelmann C, et al. Cortical demyelination and diffuse white matter injury in multiple sclerosis. Brain 2005;128:2705-2712.

13. Magliozzi R, Howell O, Vora A, et al. Meningeal B-cell follicles in secondary progressive multiple sclerosis associate with early onset of disease and severe cortical pathology. Brain 2007;130:1089-1104.

14. Simon JH, Kinkel RP, Jacobs L, Bub L, Simonian NA. Wallerian degeneration pattern in patients at risk for MS. Neurology 2000;54:1155-1160.

15. Lucchinetti CF, Popescu BF, Bunyan RF, et al. Inflammatory cortical demyelination in early multiple sclerosis. N Engl J Med 2011;365:2188-2197.

16. Gilmore CP, Donaldson I, Bo L, Owens T, Lowe J, Evangelou N. Regional variations in the extent and pattern of grey matter demyelination in multiple sclerosis: a comparison between the cerebral cortex, cerebellar cortex, deep grey matter nuclei and the spinal cord. J Neurol Neurosurg Psychiatry 2009;80:182-187.

17. Serafini B, Rosicarelli B, Franciotta D, et al. Dysregulated Epstein-Barr virus infection in the multiple sclerosis brain. J Exp Med 2007;204:2899-2912.

18. Calabrese M, Agosta F, Rinaldi F, et al. Cortical lesions and atrophy associated with cognitive impairment in relapsing-remitting multiple sclerosis. Arch Neurol 2009; 66:1144-1150.

19. Geurts JJ, Pouwels PJ, Uitdehaag BM, Polman $\mathrm{CH}$, Barkhof F, Castelijns JA. Intracortical lesions in multiple sclerosis: improved detection with 3D double inversionrecovery MR imaging. Radiology 2005;236:254-260.

20. Aloisi F, Serafini B, Magliozzi R, Howell OW, Reynolds R. Detection of Epstein-Barr virus and B-cell follicles in the multiple sclerosis brain: what you find depends on how and where you look. Brain 2010;133:e157.

21. Kappus N, Weinstock-Guttman B, Hagemeier J, et al. Cardiovascular risk factors are associated with increased lesion burden and brain atrophy in multiple sclerosis.
J Neurol Neurosurg Psychiatry Epub 2015 Feb 26. doi: 10.1136/jnnp-2014-310051.

22. Zivadinov R, Marr K, Cutter G, et al. Prevalence, sensitivity, and specificity of chronic cerebrospinal venous insufficiency in MS. Neurology 2011;77:138-144.

23. Zivadinov $\mathrm{R}$, Heininen-Brown $\mathrm{M}$, Schirda $\mathrm{CV}$, et al. Abnormal subcortical deep-gray matter susceptibilityweighted imaging filtered phase measurements in patients with multiple sclerosis: a case-control study. Neuroimage 2012;59:331-339.

24. Smith SM, Zhang Y, Jenkinson M, et al. Accurate, robust, and automated longitudinal and cross-sectional brain change analysis. Neuroimage 2002;17:479-489.

25. Benjamini Y, Drai D, Elmer G, Kafkafi N, Golani I. Controlling the false discovery rate in behavior genetics research. Behav Brain Res 2001;125:279-284.

26. Nielsen TR, Rostgaard K, Askling J, et al. Effects of infectious mononucleosis and HLA-DRB1*15 in multiple sclerosis. Mult Scler 2009;15:431-436.

27. De Jager PL, Chibnik LB, Cui J, et al. Integration of genetic risk factors into a clinical algorithm for multiple sclerosis susceptibility: a weighted genetic risk score. Lancet Neurol 2009;8:1111-1119.

28. Kooi EJ, Geurts JJ, van Horssen J, Bo L, van der Valk P. Meningeal inflammation is not associated with cortical demyelination in chronic multiple sclerosis. J Neuropathol Exp Neurol 2009;68:1021-1028.

29. Peferoen LA, Lamers F, Lodder LN, et al. Epstein Barr virus is not a characteristic feature in the central nervous system in established multiple sclerosis. Brain 2010;133: e137.

30. Tzartos JS, Khan G, Vossenkamper A, et al. Association of innate immune activation with latent Epstein-Barr virus in active MS lesions. Neurology 2012;78:15-23.

31. Angelini DF, Serafini B, Piras E, et al. Increased CD8+ T cell response to Epstein-Barr virus lytic antigens in the active phase of multiple sclerosis. PLoS Pathog 2013;9: e1003220.

32. Magliozzi R, Serafini B, Rosicarelli B, et al. B-cell enrichment and Epstein-Barr virus infection in inflammatory cortical lesions in secondary progressive multiple sclerosis. J Neuropathol Exp Neurol 2013;72:29-41.

33. Filippi M, Preziosa P, Pagani E, et al. Microstructural magnetic resonance imaging of cortical lesions in multiple sclerosis. Mult Scler 2013;19:418-426.

34. Giorgio A, Stromillo ML, Rossi F, et al. Cortical lesions in radiologically isolated syndrome. Neurology 2011;77: 1896-1899.

35. Zivadinov R, Havrdova E, Bergsland N, et al. Thalamic atrophy is associated with development of clinically definite multiple sclerosis. Radiology 2013;268: 831-841. 


\title{
Neurology \\ Neuroimmunology \& Neuroinflammation
}

\author{
Humoral response to EBV is associated with cortical atrophy and lesion burden in \\ patients with MS \\ Robert Zivadinov, Nicole Cerza, Jesper Hagemeier, et al. \\ Neurol Neuroimmunol Neuroinflamm 2016;3; \\ DOI 10.1212/NXI.0000000000000190
}

This information is current as of January 7, 2016

$\begin{array}{ll}\begin{array}{l}\text { Updated Information \& } \\ \text { Services }\end{array} & \begin{array}{l}\text { including high resolution figures, can be found at: } \\ \text { http://nn.neurology.org/content/3/1/e190.full.html }\end{array} \\ \text { Supplementary Material } & \begin{array}{l}\text { Supplementary material can be found at: } \\ \text { http://nn.neurology.org/content/suppl/2016/01/07/3.1.e190.DC1 }\end{array} \\ \text { This article cites } 30 \text { articles, } 5 \text { of which you can access for free at: } \\ \text { http://nn.neurology.org/content/3/1/e190.full.html\#\#ref-list-1 } \\ \text { This article has been cited by 3 HighWire-hosted articles: } \\ \text { http://nn.neurology.org/content/3/1/e190.full.html\#\#therarticles } \\ \text { Citations } & \begin{array}{l}\text { This article, along with others on similar topics, appears in the } \\ \text { following collection(s): }\end{array} \\ \text { MRI } & \text { http://nn.neurology.org//cgi/collection/mri } \\ \text { Multiple sclerosis } & \text { http://nn.neurology.org//cgi/collection/multiple_sclerosis } \\ & \text { Information about reproducing this article in parts (figures,tables) or in } \\ \text { its entirety can be found online at: } & \text { http://nn.neurology.org/misc/about.xhtml\#permissions } \\ \text { Permissions \& Licensing } & \text { Information about ordering reprints can be found online: } \\ \text { http://nn.neurology.org/misc/addir.xhtml\#reprintsus }\end{array}$

Neurol Neuroimmunol Neuroinflamm is an official journal of the American Academy of Neurology.

Published since April 2014, it is an open-access, online-only, continuous publication journal. Copyright $\odot$ 2016 American Academy of Neurology. All rights reserved. Online ISSN: 2332-7812.

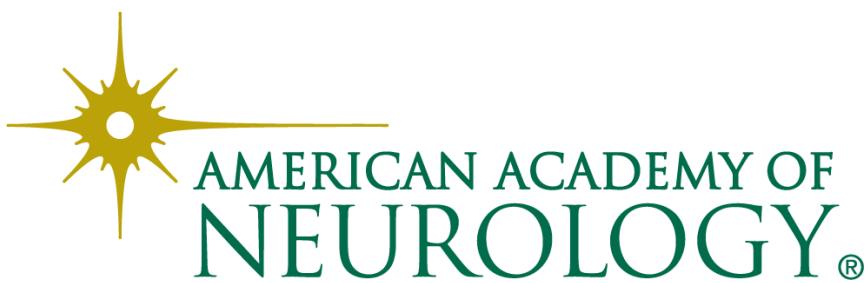

